\title{
Damped plasma waves in photoexcited plasma in semiconductors
}

\author{
Áurea R. Vasconcellos and Roberto Luzzi \\ Instituto de Física “Gleb Wataghin," Universidade Estadual de Campinas (UNICAMP), 13083-970 Campinas, São Paulo, Brazil
}

A. Sérgio Esperidião

Instituto de Física, Universidade Federal da Bahia, 40170-290 Salvador, Bahia, Brazil

(Received 11 August 1994; revised manuscript received 27 January 1995)

\begin{abstract}
We analyze the hydrodynamic modes associated with carriers in a photoinjected plasma in a directgap polar semiconductor. In this paper we concentrate our attention on the carrier material motion. Resorting to a mechanostatistical approach to irreversible thermodynamics, the coupled equations of evolution for the charge density of electrons and holes are derived. They have the form of hyperbolic equations which imply collective damped wave propagation. The kinetic coefficients on which they depend are determined at this mechanostatistical microscopic level. The corresponding hydrodynamic modes are characterized: they consist of, besides the single quasiparticle (electrons and holes) excitations, two collective modes, namely, the acoustical and optical plasma waves. Their dispersion relations and lifetimes are obtained. These hydrodynamic modes are evidenced and further characterized through the calculation and analysis of the spectrum of Raman scattering of radiation by the carriers. The motion of the charge density is analyzed and we show how the damped wave regime goes over to the Fick-like diffusive regime in the limit of very large wavelengths and very low frequencies.
\end{abstract}

\section{INTRODUCTION}

Laser light illumination of semiconductor samples in a process of photoinjection allows for the creation of a double plasma (electrons and holes in the lattice background), which is a fluid displaying hydrodynamic properties. This is the case for a semiconductor system working under far-from-equilibrium conditions, and poses interesting problems in the physics of condensed matter. In particular, as we have pointed out on other occasions, these systems provide a good testing ground for theoretical ideas in statistical thermodynamics and many-body physics, and there is also an accompanying technological interest because of their applications in electronic devices.

We consider a semiconductor sample under the action of continuous laser illumination that creates the plasma, and drives the system to a steady state in conditions far from equilibrium. Under the action of laser light of constant intensity, a uniform concentration $n$ of carriers (electrons and holes created in pairs), that increases with the laser power $I_{L}$, is produced. These carriers become internally thermalized in the subpicosecond time scale due to the strong Coulomb interaction, ${ }^{1}$ and then their macroscopic state can be characterized by a quasitemperature and quasichemical potentials. The nonequilibrium phonon system does not have a unique quasitemperature but different quasitemperatures for each mode, with a privileged distribution around an off-center region of the Brillouin zone. ${ }^{2}$

In this paper we study some hydrodynamic properties of the carrier system. We look for the evolution of local deviations in the carrier density from the homogeneous state, leaving out the question of propagation of thermal waves for consideration in the following paper. ${ }^{3}$ Hydro- dynamic theories have in large part been based on classical (sometimes called linear) irreversible thermodynamics (CIT). CIT (e.g., Ref. 4) introduces the local equilibrium assumption and leads to hydrodynamic equations which are of the parabolic type (Fourier's and Fick's diffusion equations) which predict an infinite speed of propagation for thermal and viscous signals, an unphysical feature. However, it may not be a dangerous paradox in certain situations when it becomes a good approximation, as discussed below. Furthermore, CIT does not produce good agreement with experiment in the short-wavelength and/or high-frequency regimes; examples are the case of sound absorption and its dispersion in fluids, and the description of shock waves or Mach numbers larger than $1.85 .^{5}$ Some approaches to go beyond CIT are presently available; one of them is extended irreversible thermodynamics (EIT). ${ }^{6,7}$ EIT removes the main difficulties associated with CIT, with the equations of evolution for the densities now being of the hyperbolic type, implying damped wave propagation with a finite velocity. These equations are referred to as the Maxwell-CattaneoVernotte equations. The name of Maxwell arises out of the fact that in the last century he proposed a relationship in viscous systems between forces (causes) and fluxes (effects), including relaxation processes, as contained in EIT. ${ }^{8}$ EIT can be shown to be partially encompassed in kinetic theory through alternatives to Grad's 9 moments method. ${ }^{10}$ However this kind of relationship between kinetic theory and EIT in many of its aspects has been limited to the use of a finite number of moments in Grad's method. This implies an arbitrary truncation of the set of coupled equations of evolution for the basic variables.

On the other hand, on the mechanostatistical side the so-called nonequilibrium statistical operator method 
(NSOM) seems, within the group of available formalisms, ${ }^{11}$ to offer a very powerful and practical tool. Recently it has been shown that different approaches to the NSOM, based on either heuristic or projection operator technique points of view, can be brought together under a unifying variational principle. ${ }^{12}$ This approach to the NSOM places the formalism within the scope of Jaynes' predictive statistical mechanics. ${ }^{13}$ This theory is based on the principles of Bayesian statistical inference, ${ }^{14,15}$ with a constructive criterion for setting up probability distributions, namely the maximum entropy formalism (MAXENT). ${ }^{14,16}$ The MAXENT-NSOM recovers, as special cases, equilibrium statistical mechanics, linearresponse theory, and Kubo's transport theory. ${ }^{17}$ However, the formalism is powerful enough to go beyond in order to provide mechanostatistical foundations for an irreversible thermodynamics of systems arbitrarily away from equilibrium, leading to what can be referred to as informational statistical thermodynamics (IST). ${ }^{18,19}$ IST encompasses EIT as a limiting case. Underlying ideas for the use of an informational-statistical approach after its introduction by Jaynes have been handled by several authors. One of the first approaches to IST is due to Nettleton, ${ }^{20}$ who also pointed out apparent inconsistencies in the existing phenomenological versions of EIT. ${ }^{21}$

Furthermore, the MAXENT-NSOM provides a nonlinear quantum transport theory of a large scope, which can be considered a far-reaching generalization of Mori's approach. ${ }^{22}$ In this theory the transport coefficients, which are open parameters in phenomenological thermodynamic and kinetic theories, are given at the microscopic level (that is, in terms of the underlying dynamical theory averaged with the appropriate MAXENT-NSOM statistical distribution that characterizes the nonequilibrium macroscopic state of the system), and which also incorporates nonlocality in space and time (spatial correlations and memory effects, respectively).

The hydrodynamic properties of the carrier system in photoexcited semiconductors described in this paper are based on a theory founded on the MAXENT-NSOM, that is, derived from IST. It should be noted that IST implies a generalization of Grad's moments approach, now at the quantum level and to all orders. ${ }^{18,19,23}$ Clearly, a practical use in general requires us to introduce a truncation procedure, therefore leading to an approximation that needs to be evaluated in each case, as done in the following sections. We recall that the MAXENT-NSOM is described with certain detail in Ref. 12, and the associated transport theory in Ref. 22. We have also applied the MAXENT-NSOM to the study of ultrafast relaxation processes and transport processes in highly excited plasma in semiconductors, ${ }^{24}$ where a brief review of the method is given and particularized for such cases. Of the different alternative constructions of the NSOM we use the one due to Zubarev: ${ }^{25}$ an elegant, concise, and practical approach to the formalism.

In this paper we describe the calculation of the equations of evolution of the carrier's local-charge densities. We derive those for the electron and hole equations of propagation with damping, which are of the telegraphist type arising out of the resulting Maxwell-Cattaneo-
Vernotte-like equations contained in IST. From them we are able to characterize a set of electronic elementary excitations. They are of four types: two are the excitations of single quasiparticles (electrons and holes individually), and two are collective excitations. The latter, for these charged particles, are plasma waves. One has a high frequency, and the other a low frequency and a linear energy dispersion at long wavelengths. They are termed optical and acoustical plasma waves, respectively. The existence of acoustic plasmons was predicted by Pines and co-workers ${ }^{26}$ for the case of a plasma in the solid state with two types of carriers. It should be noted that the two types of carriers in the photoinjected plasma in semiconductors we consider are produced in nonequilibrium conditions, i.e., they are generated by the continuous application of laser light. There seems to be experimental evidence of acoustic plasmons in the carrier contribution to the Raman spectra in photoexcited GaAs. ${ }^{27}$ It has been suggested, but not proved experimentally, that there is a possible presence of acoustic plasmons in the case of carriers in different valleys, ${ }^{28}$ in an ideal degenerate twocomponent (one or both superconducting) Coulomb Fermi liquid, ${ }^{29}$ and in a superconducting two-component Fermi liquid. ${ }^{30}$

The paper is organized as follows. In Sec. II, we derive the equations of evolution for the carriers' local-charge densities. They are coupled, as is the general case, ${ }^{18}$ to an infinite set of equations (the local linear momentum density and its higher-order fluxes), and then a truncation procedure is introduced as described in the text. From these equations of evolution for the carrier densities we derive the complete spectrum of elementary excitations. Further, we briefly discuss the lifetimes of these excitations, and the diffusion process of carriers. In Sec. III we calculate the electronic contribution to the Ramanscattering cross section in a typical pump-probe experiment. This is done by calculating the carrier contribution to the frequency- and wave-number-dependent dielectric function which determines the Raman spectrum. The elementary excitations are characterized by the different bands that appear in the Raman spectrum, which are related to the zeros of the real part of the dielectric function. We recover the results of the hydrodynamic analysis of Sec. II. In Sec. IV we review and discuss the results.

\section{QUASIHYDRODYNAMICS OF HEPS}

We consider an intrinsic direct-gap polar semiconductor sample illuminated by a continuous laser beam, with a power flux $I_{L}$ and a photon frequency $\omega_{L}$. The photogenerated electron-hole pairs are assumed to behave as free carriers, i.e., to be on the metallic side of the Mott transition (typically for concentrations higher than $10^{16} \mathrm{~cm}^{-3}$ ). These carriers relax energy to the lattice while their total number varies as it grows due to photon absorption but diminishes in recombination processes, until a steadystate situation is achieved when both effects are compensated. We assume a constant laser light intensity throughout the volume of laser light focalization, and stimulated emission and self-absorption are neglected in comparison with spontaneous recombination. The sys- 
tem Hamiltonian consists of the carriers' energy operator [electrons and holes in band energy states taken in the effective-mass approximation, plus the Coulomb interaction dealt with in the random-phase approximation (RPA)], the Hamiltonian of the phonon field, and the energy operators describing the interaction of carriers with lattice vibrations and with the laser and recombination radiation fields. The carrier-radiation interaction is treated in the dipole approximation, with the photon field described by a classical field incorporated in the carriers' Hamiltonian. Optical phonons are described in an Einstein (dispersionless) model. The acoustic phonons are taken as a thermal bath at temperature $T_{c}$. These and the laser source are taken as ideal reservoirs, i.e., they are assumed to remain in stationary unaltered conditions while constantly coupled with the open semiconductor sample.

Through this process of photoinjection is created a highly excited plasma in the semiconductor (HEPS) in nonequilibrium conditions. We consider the hydrodynamic properties of the carriers' system in this HEPS; and, for such a purpose, we resort to the statisticalmechanical formalism of the MAXENT-NSOM described in an earlier publication, ${ }^{31}$ where details are given. We simply note that the hydrodynamic study of the carriers' system requires us to introduce basic dynamical variables consisting of the electron density and hole density, whose Fourier amplitudes are given, respectively, by

$$
\begin{aligned}
& \hat{n}^{e}(\mathbf{Q})=\sum_{\mathbf{k}} \hat{n}_{\mathbf{k} \mathbf{Q}}^{e}=\sum_{\mathbf{k}} C_{\mathbf{k}+\mathbf{Q}}^{\dagger} C_{\mathbf{k}}, \\
& \hat{n}^{h}(\mathbf{Q})=\sum_{\mathbf{k}} \hat{n}_{\mathbf{k Q}}^{h}=\sum_{\mathbf{k}} h_{-\mathbf{k}-\mathbf{Q}} h_{-\mathbf{k}}^{\dagger},
\end{aligned}
$$

where $c\left(c^{\dagger}\right)$ and $h\left(h^{\dagger}\right)$ are annihilation (creation) operators in electron and hole states, as well as those for the linear momenta

$$
\hat{\mathbf{p}}^{e(h)}(\mathbf{Q})=\sum_{\mathbf{k}} \hbar\left(\mathbf{k}+\frac{1}{2} \mathbf{Q}\right) \hat{n}_{\mathbf{k} \mathbf{Q}}^{e(h)}
$$

and all other higher-order (tensorial) fluxes of matter.

Quantities of Eqs. (1) and (2) are relevant for the study of the material motion of the system. For the analysis of the thermal motion we need to introduce the energy density

$$
\hat{\varepsilon}_{\alpha}(\mathbf{Q})=\sum_{\mathbf{k}} \frac{\hbar^{2}}{2 m_{\alpha}^{*}}\left[\mathbf{k} \cdot(\mathbf{k}+\mathbf{Q})+\frac{1}{2} Q^{2}\right] \hat{n}_{\mathbf{k Q}}^{\alpha},
$$

the energy flux

$$
\begin{aligned}
\hat{\mathbf{I}}_{\alpha}(\mathbf{Q})=\sum_{\mathbf{k}} \frac{\hbar^{2}}{2 m_{\alpha}^{*}}\left[\mathbf{k} \cdot(\mathbf{k}+\mathbf{Q})+\frac{1}{2} Q^{2}\right] \\
\times \frac{\hbar}{m_{\alpha}^{*}}\left(\mathbf{k}+\frac{1}{2} \mathbf{Q}\right) \hat{n}_{\mathbf{k} \mathbf{Q}}^{\alpha},
\end{aligned}
$$

and all the other higher-order (tensorial) fluxes of energy. In Eqs. (3) and (4) $\alpha=e$ for electrons and $\alpha=h$ for holes.

Material and thermal motion are coupled together in the equations of evolution for corresponding macrovariables. The coupling occurs through cross-kinetic terms associated with thermoelectriclike effects. We now consider both types of motion separately, in order to concentrate attention alternately on plasma and thermal waves, respectively, to facilitate their study and a better appreciation of their physical characteristics. To neglect the coupling effects only produces minor numerical differences in the characterization of the hydrodynamical modes. In fact, they give rise to frequency shifts and additional contributions to the lifetimes. This has been tested; we omit the lengthy details for the sake of brevity, but call attention to the result presented in Sec. III where, for a connection with Raman experiments, a complete calculation is performed which implies the inclusion of both mass and energy densities and their fluxes of all orders: as noted there, corrections to the frequencies and lifetimes obtained in the approximate calculation are-for the case of GaAs considered-of the order of $20 \%$. In the present paper we consider the material motion (plasma waves), and in our following paper the thermal motion (heat propagation). ${ }^{3}$

Consequently we next consider the derivation of the equations of evolution for the density and all its fluxes. A practical use of the method requires us to introduce a truncation procedure in this infinite set of coupled integrodifferential equations: we choose the contracted set consisting of the carriers' density and linear momenta, namely

$$
\begin{aligned}
& n^{e(h)}(\mathbf{Q}, t)=\operatorname{Tr}\left\{\hat{n}^{e(h)}(\mathbf{Q}) \bar{\rho}(t, 0)\right\}, \\
& \mathbf{p}^{e(h)}(\mathbf{Q}, t)=\operatorname{Tr}\left\{\hat{\mathbf{p}}^{e(h)}(\mathbf{Q}) \bar{\rho}(t, 0)\right\},
\end{aligned}
$$

where $\bar{\rho}(t, 0)$ is the corresponding auxiliary nonequilibrium statistical operator in MAXENT-NSOM, ${ }^{12,25}$ which in the present case takes the generalized nonequilibrium Gibbsian-like form given by Eq. (18) in Ref. 31.

A contracted description of this type, i.e., here in terms of a density and its flux, is equivalent to a truncated description in the classical Grad's moments approach, ${ }^{9}$ as shown in Ref. 18. The approximation needs to be evaluated in terms of some appropriate expansion parameter. As discussed elsewhere, ${ }^{18}$ in this case it is $\gamma=\left(\bar{v} Q / \omega_{\mathrm{pl}}\right)^{2}$, where $\bar{v}$ is the average velocity of the carriers; $Q=2 \pi / \lambda$, where $\lambda$ is the wavelength of the excitation; and $\omega_{\mathrm{pl}}$ the optical plasma frequency. In what follows we shall keep only linear terms in the dispersion relations, implying a long-wavelength limit characterized by $\gamma \ll 1$.

The equations of evolution for the basic set of variables of Eqs. (5) follow from application of the MAXENTNSOM statistical method as described in Ref. 22, and are given by Eqs. (32a) $-(32 d)$ in Ref. 31 . We recall that in their derivation space correlations were neglected; that is, they are equations local in space, and linearized in the inhomogeneities. Thus, we consider small local derivations from the homogeneous state.

In continuation we consider the homogeneous state of reference as stationary, which is a result of the application of cw laser illumination. Consequently, the kinetic coefficients in Eqs. (32) in Ref. 31 are constant in time. These four equations, which are of first order in the time derivative of the four basic variables, are next transformed into two equations, albeit of second order in the time derivative, for the densities. For that purpose we derive with respect to time in Eqs. (32a) and (32c) in Ref. 31 , and next the dependence on the momenta is 
eliminated through the use of Eqs. (32b) and (32d), to finally obtain the following set of coupled equations, namely

$$
\begin{aligned}
& \frac{1}{c_{e}^{2}} \frac{\partial^{2}}{\partial t^{2}} n^{e}(\mathbf{Q}, t)+\frac{1}{D_{e}} \frac{\partial}{\partial t} n^{e}(\mathbf{Q}, t)-\frac{1}{D_{\mathrm{eh}}} \frac{\partial}{\partial t} n^{h}(\mathbf{Q}, t)+Q^{2} n^{e}(\mathbf{Q}, t)+\alpha^{\mathrm{eh}}(\mathbf{Q}) Q^{2}\left[n^{e}(\mathbf{Q}, t)+n^{h}(\mathbf{Q}, t)\right]=0 \\
& \frac{1}{c_{h}^{2}} \frac{\partial^{2}}{\partial t^{2}} n^{h}(\mathbf{Q}, t)+\frac{1}{D_{h}} \frac{\partial}{\partial t} n^{h}(\mathbf{Q}, t) \frac{1}{D_{\mathrm{he}}} \frac{\partial}{\partial t} n^{e}(\mathbf{Q}, t)+Q^{2} n^{h}(\mathbf{Q}, t)+\alpha^{h e}(\mathbf{Q}) Q^{2}\left[n^{e}(\mathbf{Q}, t)+n^{h}(\mathbf{Q}, t)\right]=0
\end{aligned}
$$

where

$$
\begin{aligned}
& c_{e(h)}^{2}=\frac{2}{3} m_{e(h)}^{-1} \sum_{\mathbf{k}} \epsilon_{\mathbf{k}}^{e(h)} f_{\mathbf{k}}^{e(h)}\left[1-f_{\mathbf{k}}^{e(h)}\right], \\
& D_{e}^{-1}=\left[\gamma^{\mathrm{he}}+\widetilde{\Gamma}^{\mathrm{he}}+\widetilde{\Lambda}^{e}\right] / c_{e}^{2}, \\
& D_{h}^{-1}=D_{e}^{-1} \text { with exchange } e \leftrightarrow h, \\
& D_{\mathrm{eh}}^{-1}=\left[\gamma^{\mathrm{eh}}+\frac{m_{h}}{m_{e}} \widetilde{\Gamma}^{\mathrm{eh}}\right] / c_{e}^{2}, \\
& D_{\mathrm{he}}^{-1}=D_{\mathrm{eh}}^{-1} \quad \text { with exchange } e \leftrightarrow h, \\
& \alpha^{\mathrm{eh}}(\mathbf{Q})=V(Q) n_{e} / m_{e} c_{e}^{2}=\omega_{\mathrm{pl}, e} / Q^{2} c_{e}^{2}, \\
& \alpha^{\mathrm{he}}(\mathbf{Q})=\alpha^{\mathrm{eh}} \quad \text { with exchange } e \leftrightarrow h,
\end{aligned}
$$

with all quantities on the right of Eqs. (7) defined in Ref. 31 , and $\omega_{\mathrm{pl}, e(h)}^{2}=4 \pi n_{e(h)} e^{2} / m_{e(h)}$.

In Eqs. (6) above we have only taken terms involving the densities when associated with Coulomb interaction (last term on the left), neglecting, when compared with it, additional terms also proportional to the densities but associated with interactions with the phonon and radiation fields.

It should be noted that Eqs. (6) are space-Fourier transforms of a telegraphistlike equation with a source (the term involving Coulomb interaction). Hence Eqs. (6) correspond to damped propagations of oscillations whose frequencies (dispersion relations) are given by the corresponding characteristic equation, namely

$$
\begin{gathered}
{\left[\omega^{2}-i \frac{c_{e}^{2}}{D_{e}} \omega+c_{e}^{2}\left(1+\alpha^{\mathrm{eh}}\right) Q^{2}\right]\left[\omega^{2}-i \frac{c_{h}^{2}}{D_{h}}+c_{h}^{2}\left(1+\alpha^{\mathrm{he}}\right) Q^{2}\right]} \\
-\left[i \frac{c_{e}^{2}}{D_{\mathrm{he}}} \omega+c_{e}^{2}\left(1+\alpha^{\mathrm{he}}\right) Q^{2}\right]\left[i \frac{c_{h}^{2}}{D_{\mathrm{eh}}}+c_{h}^{2} \alpha^{\mathrm{he}} Q^{2}\right]=0 .
\end{gathered}
$$

Next we look for the dispersion relations in the absence of damping (i.e., all $D^{-1}$ set equal to zero), and in lowest order in $Q$ (we neglect $\alpha_{\mathrm{eh}} \alpha_{\mathrm{he}} Q^{4}$ ). Two cases can be proved: (i) neglecting the effects of Coulomb interaction $(\alpha=0)$, we obtain the excitation energy of the single quasiparticles; and (ii) including the effects of Coulomb interactions we obtain two collective (plasma) excitations. In fact, in case (i) in the highly degenerate regime we find

$$
\omega_{e}=v_{e F} Q, \omega_{h}=v_{h F} Q,
$$

where $v_{e(h) F}$ is the Fermi velocity of electrons (holes). These are the averages over the ensemble of the single quasiparticle excitations of $\epsilon_{\mathbf{k}+Q}^{e(h)}-\epsilon_{\mathbf{k}}^{e(h)} \simeq \hbar \mathbf{k} \cdot \mathbf{Q} / m_{e(h)}$. In case (ii) we find the characteristic eigenfrequencies

$$
\omega_{\mathrm{pl}, \mathrm{op}}=\left(4 \pi n e^{2} / m_{x}\right)^{1 / 2}, \omega_{\mathrm{pl}, \mathrm{Ac}}=s_{A} Q,
$$

where $s_{A}=\left(v_{e F} v_{h F} / 3\right)^{1 / 2}$ is the group velocity of the latter.

The two frequencies of Eq. (10) are (1) $\omega_{\mathrm{pl} \text {,op }}$ (the dispersionless frequency in the given approximation of small $Q$ ) of propagation of the usual plasma wave corresponding to the collective motion, in the relative coordinate, of electron-hole pairs interacting through the bare Coulomb interaction, and called the optical plasma mode; and (2) $\omega_{\mathrm{pl}, \mathrm{Ac}}$, with a linear dispersion relation, which can be ascribed to the acoustical plasma mode conjectured by Pines. ${ }^{26}$ The latter can be interpreted as arising out of the collective motion of the centers of mass of the electron-hole pairs, with the particles interacting through the screened part of Coulomb interaction. In fact, if for the plasma frequency we take the expression ${ }^{26}$

$$
\omega^{2}=(n / m) Q^{2} V(Q),
$$

(1) $\omega_{\mathrm{pl}, \mathrm{op}}$ follows for $m=m_{x}$, and $V(Q)$, the bare Coulomb interaction, is $4 \pi e^{2} / Q^{2} \varepsilon_{0}$; and (2) $\omega_{\mathrm{pl}, \mathrm{Ac}}$ follows for $m=\boldsymbol{M}=m_{e}+m_{h}$, and the screened Coulomb interaction $V(Q)=4 \pi e^{2} /\left(Q^{2}+\Lambda_{\mathrm{FT}}^{-2}\right)$, after taking $Q \simeq 0$ and $\Lambda_{\text {FT }}$ as the Fermi-Thomas screening length (namely, $\Lambda_{F}^{-2}=4 \pi n e^{2}\left(g_{F e}+g_{F h}\right)$, with $g_{F e(h)}=\frac{3}{2} \epsilon_{F e(h)}$ being the density of states at the Fermi level of energy $\left.\epsilon_{\mathrm{Fe}(h)}\right)$ : then $\omega^{2}=\hbar^{2} Q^{2}\left(3 \pi^{2} n\right)^{2 / 3} / 3 M m_{x}$, recovering the expression $\omega_{\mathrm{pl}, \mathrm{Ac}}^{2}=v_{F e} v_{F h} Q^{2} / 3$.

Once the collective oscillations have been characterized, we next look for their lifetimes, to be provided by the complete solution of Eq. (8). We consider the limit of weak dissipation of the waves, and then we write $\omega_{\mathrm{op}}=\omega_{\mathrm{pl}, \mathrm{op}}+i \delta_{\mathrm{op}}$ and $\omega_{\mathrm{Ac}}=\omega_{\mathrm{pl}, \mathrm{Ac}}+i \delta_{\mathrm{Ac}}$, and we solve for $\delta$ in Eq. (8) up to first order in that quantity. We find that

$$
\begin{aligned}
& \delta_{\mathrm{op}} \simeq \frac{1}{2} {\left[\widetilde{\Lambda}^{e} \frac{\omega_{\mathrm{pl}, e}^{2}}{\omega_{\mathrm{pl}, \mathrm{op}}^{2}}+\widetilde{\Lambda}^{h} \frac{\omega_{\mathrm{pl}, h}^{2}}{\omega_{\mathrm{pl}, \mathrm{op}}^{2}}\right], } \\
& \delta_{\mathrm{Ac}} \simeq \frac{1}{2}\left[c_{e}^{2}\left(D_{e}^{-1}+D_{\mathrm{eh}}^{-1}\right) \frac{\omega_{\mathrm{pl}, e}^{2}}{\omega_{\mathrm{pl}, \mathrm{Ac}}^{2}}\right. \\
&\left.+c_{h}^{2}\left(D_{h}^{-1}+D_{\mathrm{he}}^{-1}\right) \frac{\omega_{\mathrm{pl}, h}^{2}}{\omega_{\mathrm{pl}, \mathrm{Ac}}^{2}}\right] .
\end{aligned}
$$

Inspection of Eqs. (12) shows us that the lifetime of the optical plasmons is determined exclusively by interaction with the phonon field. The lifetime of the acoustic plasmons is also determined by the interaction of the carriers with the laser and recombination radiation fields, 
which appear in the direct and cross-diffusion coefficients, respectively $D_{e(h)}$ and $D_{\text {eh(he) }}$ defined in Eqs. (7). It ought to be stressed that, since $\omega_{\mathrm{pl}, \mathrm{Ac}}=s_{A} q$, Eq. (12b), derived on the basis that $\delta_{\mathrm{Ac}} \ll \omega_{\mathrm{pl}, \mathrm{Ac}}$, is only valid for not too small values of $Q$. This tells us that when going from high values of $Q$ to small values of $Q$, this collective movement changes in character from a damped oscillation to a nonoscillating overdamped regime. This will be better shown in the study of Raman scattering in Sec. III.

After characterizing hydrodynamic modes associated with the coupled material motion of electrons and holes in HEPS, let us close this section with an analysis of the evolution of the local deviations of the whole charge. Considering charge neutrality in the movement, i.e., setting $n^{e}(\mathbf{Q}, t)=n^{h}(\mathbf{Q}, t)$, we find for the charge density $n(\mathbf{Q}, t)=n^{e}(\mathbf{Q}, t)+n^{h}(\mathbf{Q}, t)$, after summation of Eqs. (6), that

$\frac{1}{c^{2}} \frac{\partial^{2}}{\partial t^{2}} n(\mathbf{Q}, t)+\frac{1}{D} \frac{\partial}{\partial t} n(\mathbf{Q}, t)+Q^{2} n(\mathbf{Q}, t)=-A n(\mathbf{Q}, t)$,

or, in direct space,

$$
\frac{1}{c^{2}} \frac{\partial^{2}}{\partial t^{2}} n(\mathbf{r}, t)+\frac{1}{D} \frac{\partial}{\partial t} n(\mathbf{r}, t)-\nabla^{2} n(\mathbf{r}, t)=-A n(\mathbf{r}, t) \text {. }
$$

We recall that the dependence on $\mathbf{Q}$ of the coefficients has been neglected, leading to the local space formulation given by Eq. (14) which is also local in time as a result of the second-order approximation in relaxation theory (SOART) approximation we used. In Eqs. (13) and (14) we introduced the coefficients

$$
\begin{aligned}
& c^{-2}=\frac{1}{2}\left[c_{e}^{-2}+c_{h}^{-2}\right], \\
& D^{-1}=\frac{1}{2}\left[D_{e}^{-1}+D_{h}^{-1}-D_{\mathrm{eh}}^{-1}-D_{\mathrm{he}}^{-1}\right], \\
& A=\left(\omega_{\mathrm{pl}, e}^{2} / c_{e}^{2}\right)+\left(\omega_{\mathrm{pl}, h}^{2} / c_{h}^{2}\right) .
\end{aligned}
$$

Equation (14) is an equation of the telegraphist type (the left side) with (on the right side) a term directly proportional to the local deviation of the charge density: let us recall that it is a consequence of the Coulomb interaction that is responsible for the emergence of the collective motion. Hence Eq. (14) implies the propagation of damped waves with velocity $c$, and a lifetime related to quantity $D$ which plays the role of an ambipolar diffusion coefficient. $^{31}$

This ambipolar diffusion coefficient depends on the interaction of carriers with phonons and the recombination radiation field; the effect of the laser field disappears in the differences of gamma coefficients once the dipolar approximation and the limit of $Q$ going to zero are taken. In ultrafast pump-probe experiments, during tenfold to hundredfold picosecond time intervals, the diffusion of carriers out of the region of the volume of laser illumination is shown to be important for the macroscopic characterization of HEPS. ${ }^{32}$ In this situation the effect of the interaction with phonons predominates over the effects of recombination, which becomes relevant only on the nanosecond time scale. In stationary conditions, resulting from continuous laser illumination, considered in deriving the equation of charge-density propagation, Eq. (14), both types of contributions are present; that is, those arising from carrier-phonon collisions, responsible for the presence of coefficients $\tilde{\Lambda}$, and interaction with the recombination radiation field, responsible for coefficients $\gamma$ and $\widetilde{\Gamma}$. In the case of $\mathrm{cw}$ laser illumination the contribution to the dissipation arising out of recombination effects is much larger than that due to electron-phonon interaction. ${ }^{33}$ This is a result that in the stationary state almost immediately follows internal thermalization of carriers and phonons. A study of diffusion in HEPS is reported in Ref. 31 .

Equation (14) governs the evolution of the charge carrier density which, as noted, implies a damped undulatory motion. It is composed of the superposition of movements associated with different modes previously characterized in the analysis of Eqs. (6). Let us next look to some limiting cases. In the very long-wavelength limit $(Q \rightarrow 0)$ and in the degenerate state when $c_{e} \sim v_{e F} \gg c_{h} \sim v_{h F}$, the motion is dominated by the optical plasma wave, with frequency $\omega_{\mathrm{pl}, \mathrm{op}} \simeq \omega_{\mathrm{pl}, e}$ and a lifetime $\sim D / v_{e F}^{2}$. Consider now the limit of infinite velocity of propagation $c$, while $D$ and $A$ are kept finite. In this limit Eq. (14) becomes

$$
\left[\frac{\partial}{\partial t}-D \nabla^{2}\right] n(\mathbf{r}, t)=-D A n(\mathbf{r}, t),
$$

which is Fick's diffusion equation but with an extra term on the right. The latter can be written as $-n(\mathbf{r}, t) / \tau_{c}$, with $\tau_{c}=(D A)^{-1}$, to be interpreted as a decaying term for the local deviation from homogeneity of the charge density, forced by the Coulomb interaction in order to damp out the increase in energy associated with the charge-density-wave formation. To obtain this result the limit we took seems somewhat arbitrary, but it follows that it is equivalent, that is, it also follows Eq. (16), if one takes $\partial \mathbf{p}^{e(h)} / \partial t=0$. This means considering a quasistatic regime, or a uniform motion in the sense that the rate of variation of the momenta is null. In such a limit we go from the regime of validity of EIT, with hyperbolic equations of evolution and a damped wave motion, to the regime of LIT, with parabolic equations of evolution and a diffusive motion (implying propagation of thermal and viscous perturbations with infinite velocity). We also call attention to the fact that Eq. (16) follows approximately in the overdamped regime of Eq. (14), i.e., when $\omega<<c^{2} / D$; that is, in the very low-frequency limit.

In Sec. III we extend the study of the elementary excitations in HEPS presented in this section, looking for their characterization in the Raman-scattering cross section.

\section{THE RAMAN-SCATTERING CROSS SECTION}

Raman spectra provide information on the spectrum of elementary excitations in semiconductors, ${ }^{34}$ and then we resort to a calculation of the contribution of the carriers 
in order to characterize the elementary excitations in HEPS we derived and discussed in Sec. II. First we note that the scattering cross section is given in terms of the imaginary part of the reciprocal of the frequency and wave-vector-dependent dielectric function, ${ }^{34}$ i.e.,

$$
\frac{d^{2} \sigma}{d \Omega d \omega} \sim\left[1-e^{-\beta \hbar \omega}\right]^{-1} \operatorname{Im} \varepsilon^{-1}(\mathbf{Q}, \omega),
$$

where $\hbar \omega$ is the energy transfer and $\hbar \mathbf{Q}$ the momentum transfer in the scattering event. The dielectric function can be calculated using the fact that, according to Maxwell's equations,

$$
\varepsilon^{-1}(\mathbf{Q}, \omega)=1+n(\mathbf{Q}, \omega) / r_{0},
$$

where -en $(\mathbf{Q}, \omega)$ is the Fourier transform (in time and space) of the charge density that arises in the system under the action of a probing charge of the form $-e r_{0} \exp (-i \omega t+\mathbf{Q} \cdot \mathbf{r})$; hence we need to calculate $n(\mathbf{Q}, \omega)$ in the nonequilibrium HEPS. For that purpose we improve upon the truncation procedure used in Sec. II, introducing as basic variables the nondiagonal elements of the single-particle density matrix, namely

$$
\begin{aligned}
& n_{\mathrm{kQ}}^{e}(t)=\operatorname{Tr}\left\{\hat{n}_{\mathrm{kQ}} \rho^{e}(t, 0)\right\}=\operatorname{Tr}\left\{c_{\mathrm{k}+\mathrm{Q}^{c}}^{\dagger} \overline{\mathrm{\rho}}(t, 0)\right\}, \\
& n_{\mathrm{kQ}}^{h}(t)=\operatorname{Tr}\left\{\hat{n}_{\mathrm{kQ}}^{h} \rho(t, 0)\right\}=\operatorname{Tr}\left\{h_{-\mathrm{k}-\mathrm{Q}} h_{-\mathrm{k}}^{\dagger} \bar{\rho}(t, 0)\right\},
\end{aligned}
$$

and then the auxiliary NSO is taken to be

$$
\begin{aligned}
\bar{\rho}(t, 0)= & \exp \left\{-\phi(t)-\beta_{c}\left[H_{c}-\mu_{e} N_{e}-\mu_{h} N_{h}\right]\right. \\
& \left.-\sum_{\mathbf{q} \mu} F_{\mathrm{q} \mu} \widehat{v}_{\mathrm{q} \mu}-\sum_{\mathrm{kQ}}\left[F_{\mathrm{kQ}}^{e}(t) \hat{n}_{\mathrm{kQ}}^{e}+F_{\mathrm{kQ}}^{h}(t) \hat{n}_{\mathrm{kQ}}^{h}\right]\right\} .
\end{aligned}
$$

In Eq. (20), $F_{\mathrm{kQ}}^{e(h)}$ are the nonequilibrium thermodynamic coefficients conjugated to the quantities of Eqs. (19), and the homogeneous part is taken as stationary. We stress the fact that the density and linear momentum variables $n^{e(h)}(\mathbf{Q}, t)$ and $\mathbf{p}^{e(h)}(\mathbf{Q}, t)$ of Sec. II are linear combinations of those of Eqs. (19), and also that $\bar{\rho}$ of Eq. (20) reproduces that of Ref. 31 if we impose the choice (truncation)

$$
F_{\mathbf{k Q}}^{e(h)}(t)=-\beta(t) \mu^{e(h)}(\mathbf{Q}, t)-\beta(t) \mathbf{v}^{e(h)}(\mathbf{Q}, t) \cdot \hbar \mathbf{k} .
$$

Thus the basic set of variables we use in this section implies a large enhancement of those in Sec. II. We stress that in this way we are dealing here with an approach that can be considered equivalent to including an infinite set of moments in Grad's formalism. It is possible to obtain a nearly exact expression for the dielectric function in this case.

We derived the equations of motion for the variables of Eq. (19) in SOART, and linearized in those variables, to obtain

$$
\begin{aligned}
i \hbar \frac{\partial}{\partial t} n_{\mathrm{kQ}}^{e}(t)= & V(\mathbf{Q}) \Delta f_{\mathrm{kQ}^{r}}^{e} r_{0}+\Delta E_{\mathrm{kQ}}^{e} n_{\mathbf{k Q}}^{e}(t) \\
& -2 V(\mathbf{Q}) \Delta f_{\mathrm{kQ}}^{e}\left[n^{e}(\mathbf{Q}, t)+n^{h}(\mathbf{Q}, t)\right] \\
& +i \bar{B}_{\mathbf{k Q}}^{\mathrm{eh}} n_{\mathrm{kQ}}^{h}(\grave{t})-i \bar{B}_{\mathbf{k Q}}^{\mathrm{ee}} n_{\mathrm{kQ}}^{e}(t)
\end{aligned}
$$

$$
i \hbar \frac{\partial}{\partial t} n_{\mathrm{kQ}}^{h}(t)=i \hbar \frac{\partial}{\partial t} n_{\mathrm{kQ}}^{e}(t) \text { with exchange } e \leftrightarrow h
$$

where

$$
\begin{aligned}
& \Delta E_{\mathrm{kQ}}^{e(h)}=\epsilon_{\mathrm{k}+\mathrm{Q}}^{e(h)}-\epsilon_{\mathrm{k}}^{e(h)}, \\
& \epsilon_{\mathrm{k}}^{e}=E_{G}+\hbar^{2} k^{2} / 2 m_{e}, \\
& \epsilon_{\mathrm{k}}^{h}=\hbar^{2} k^{2} / 2 m_{h}, \\
& \Delta f_{\mathrm{kQ}}^{e(h)}=f_{\mathrm{k}+\mathrm{Q}}^{e(h)}-f_{\mathrm{k}}^{e(h)}, \\
& V(\mathbf{Q})=4 \pi e^{2} / \varepsilon_{0} V Q^{2},
\end{aligned}
$$

and $f_{\mathbf{k}}^{e^{(h)}}$ are distribution functions (see Ref. 31) for electrons (holes). We recall that the Coulomb interaction has been dealt with in the RPA. Furthermore, coefficients $B$ are related to relaxation effects resulting from the interaction of the carriers with the laser and recombination radiation fields, while the interaction with the phonons is neglected since it produces effects much smaller than those generated by the others, and then

$$
\begin{aligned}
& \bar{B}_{\mathrm{kQ}}^{\mathrm{eh}}=\bar{B}_{\mathrm{kQ}}^{\mathrm{hh}}=\hbar B_{\mathrm{k}}^{\mathrm{eh}}+\hbar B_{\mathrm{k}+\mathrm{Q}}^{\mathrm{eh}}, \\
& \bar{B}_{\mathrm{kQ}}^{\mathrm{he}}=\bar{B}_{\mathrm{kQ}}^{\mathrm{ee}}=\hbar B_{\mathrm{k}}^{\text {he }}+\hbar B_{\mathrm{k}+\mathrm{Q}}^{\text {eh }},
\end{aligned}
$$

where coefficients $B$ after the last equal sign are defined in Ref. 31 .

Fourier transforming the time variables in Eqs. (22) reduces them to a coupled set of algebraic equations which are solved, and the result is used to obtain $n(\mathbf{Q}, \omega)$, which once replaced in Eq. (18) leads to the expression

$$
\varepsilon(\mathbf{Q}, \omega)=1-V(\mathbf{Q}) \sum_{\mathbf{k}} N(\mathbf{k}, \mathbf{Q} ; \omega) D^{-1}(\mathbf{k}, \mathbf{Q} ; \omega),
$$

where

$$
\begin{aligned}
N(\mathbf{k}, \mathbf{Q} ; \omega)= & \left(\Delta f_{\mathrm{kQ}}^{e}-\Delta f_{\mathrm{kQ}}^{h}\right)\left[\hbar \omega-i\left(\bar{B}_{\mathrm{kQ}}^{\mathrm{eh}}+\bar{B}_{\mathrm{kQ}}^{\mathrm{he}}\right)\right] \\
& -\left(\Delta f_{\mathrm{kQ}}^{e} \Delta E_{\mathrm{kQ}}^{h}+\Delta f_{\mathrm{kQ}}^{h} \Delta E_{\mathrm{kQ}}^{e}\right) \\
D(\mathbf{k}, \mathbf{Q} ; \omega)= & \left(\hbar \omega+\Delta E_{\mathrm{kQ}}^{e}-i \bar{B}_{\mathrm{kQ}}^{\mathrm{he}}\right)\left(\hbar \omega-\Delta E_{\mathrm{kQ}}^{h}-i \bar{B}_{\mathrm{kQ}}^{\mathrm{eh}}\right) \\
& +\bar{B}_{\mathrm{kQ}}^{\mathrm{eh}} \bar{B}_{\mathrm{kQ}}^{\mathrm{he}} .
\end{aligned}
$$

Equation (25) provides an expression for the RPA dielectric function of the electron-hole system in the homogeneous and steady state in the photoinjected plasma in HEPS. In the limit of vanishing coefficients $B$, we recover the well-known Lindhardt (RPA) dielectric function for the system of electron and hole fluids.

To perform numerical calculations one needs to specify the system and the experimental conditions. We choose the case of GaAs illuminated by laser light with photon energy $\hbar \omega_{L}=3.4 \mathrm{eV}$, while kept at very low temperatures, i.e., $\beta^{-1}$ much smaller than the Fermi temperature of electrons and holes, so that the distribution functions $f_{\mathbf{k}}^{e(h)}$, that are of the Fermi-Dirac type in the internally 


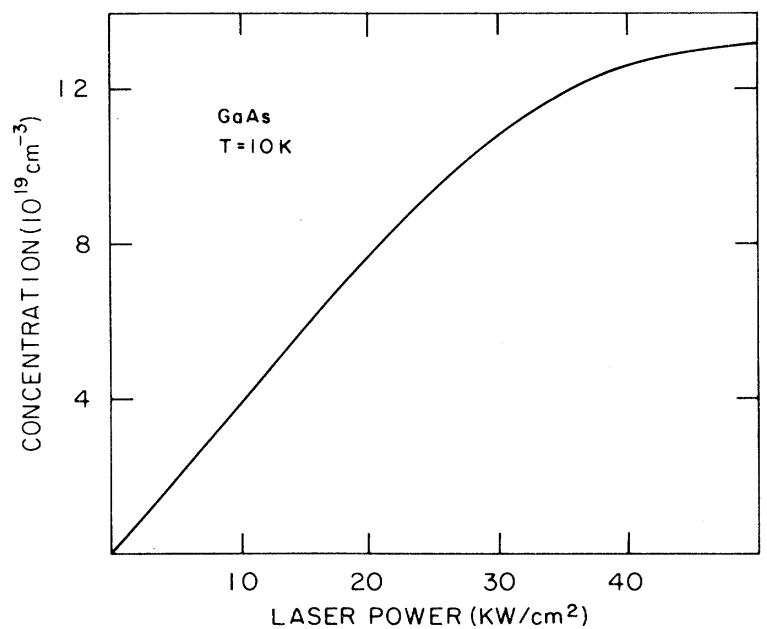

FIG. 1. The density of photoinjected carriers as a function of the laser power (cf. Ref. 32).

thermalized carrier system, can be approximated by step functions, the step limit being the Fermi energy. The laser power is taken to be $\sim 2.5 \mathrm{~kW} / \mathrm{cm}^{2}$, which, according to Ref. 33, produces a photogenerated concentration $n \simeq 10^{19} \mathrm{~cm}^{-3}$ (see Fig. 1).

Figure 2 shows the Raman spectrum for $Q=10^{4} \mathrm{~cm}^{-1}$, and Fig. 3 the real part of the dielectric function. The three bands in the Raman spectrum correspond, as expected, to the three types of excitations discussed in Sec. II, namely from lower to higher frequencies, the one due to scattering by hole individual excitations, the one due to acoustic plasmons, and the one due to electron individual excitations; the fourth one, due to optical plasmons (the highest infrequency), is left out of the range of frequencies in the figure. The frequencies at the

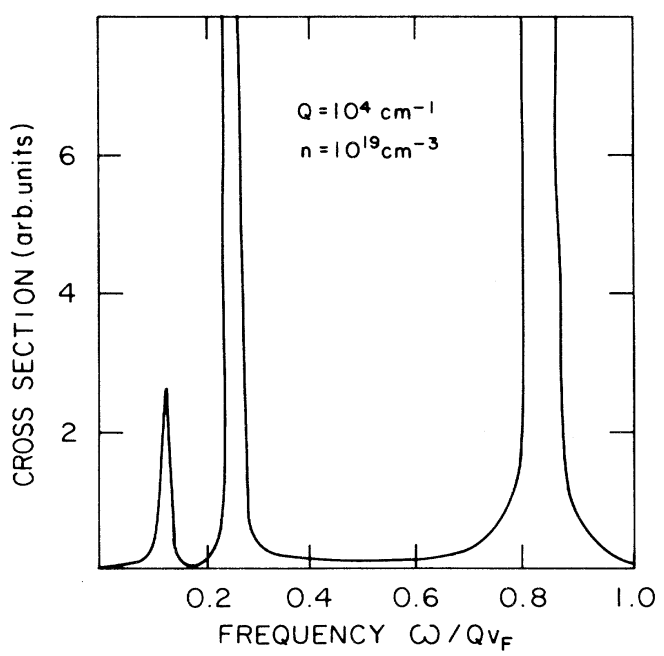

FIG. 2. The Raman spectrum for the cases $n=10^{19} \mathrm{~cm}^{-3}$ and $Q=10^{4} \mathrm{~cm}^{-1} . v_{F}$ is the Fermi velocity of the electrons.

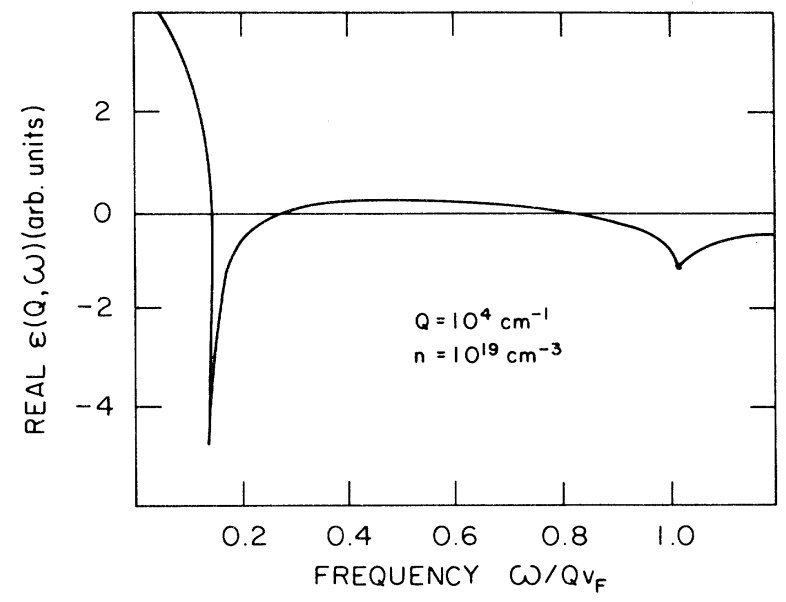

FIG. 3. The real part of the frequency and wave-vectordependent dielectric function for $n$ and $Q$ as given in the caption to Fig. 2.

positions of the peaks of the bands correspond very nearly to the frequencies at which the real part of the dielectric function is zero. The width of the band due to scattering by the plasma wave is a result of the lifetime of this excitation. There is a good agreement with results obtained in Sec. II; the frequency at the peak of the Raman line corresponding to scattering by the acoustic plasma wave is $\sim 0.24 Q v_{e F}$, and from Eq. (10) we find $0.2 Q v_{e F}$. The linewidth is $\sim 0.03 Q v_{e F}$, and so $\delta_{\mathrm{Ac}} /$ $\omega_{\mathrm{pl}, \mathrm{Ac}} \sim 0.1$, and the excitation corresponds to a weakly damped wave with wave number $10^{4} \mathrm{~cm}^{-1}$.

Figure 4 shows the Raman spectrum for several values of $Q$, which indicates a tendency - reinforced when taking ever smaller values of $Q$ - to a large overlap of the

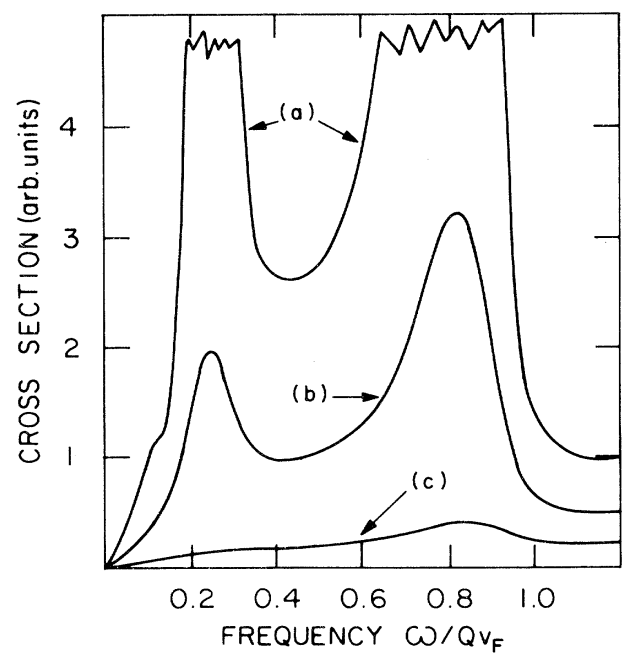

FIG. 4. The Raman spectrum for the cases $n=10^{19} \mathrm{~cm}^{-3}$ and (a) $Q=50 \mathrm{~cm}^{-1}$, (b) $25 \mathrm{~cm}^{-1}$, and (c) $10 \mathrm{~cm}^{-1}$. 


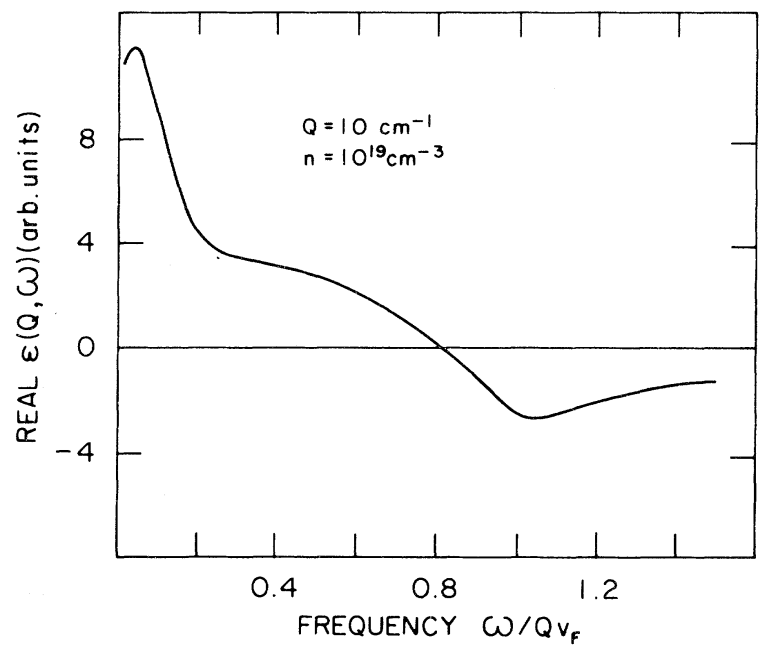

FIG. 5. The real part of the frequency and wave-vectordependent dielectric function for $n=10^{19} \mathrm{~cm}^{-3}$ and $Q=10$ $\mathrm{cm}^{-1}$.

three bands. In the curve for the real part of the dielectric function, for $Q=10 \mathrm{~cm}^{-1}$, shown in Fig. 5, we see that the two zeros at low frequency are no longer present, being replaced by a dip at $\omega / Q v_{F} \sim 0.25$. At very low wave numbers there is no oscillatory motion, in accord with what was stated in Sec. II; that is, the excitation is associated with an overdamped motion.

Hence an analysis of the Raman scattering spectrum shows the four types of elementary excitations in the system, namely the two due to scattering by quasiparticles and the two due to the acoustic and optical plasma waves. The frequency and lifetime of the plasma waves are determined from the Raman lines, which were derived-we recall-in an exact calculation of the dielectric function in Landau's quasiparticle approach. When they are compared with results obtained resorting to the truncated procedure used in Sec. II, the numerical differences are within the order of $20 \%$.

\section{CONCLUDING REMARKS}

We have presented a study of the hydrodynamic properties of the double Fermi liquid of electrons and holes in a plasma in a semiconductor generated by intense laser illumination. To deal with this far-from-equilibrium system we resorted to the concise and seemingly powerful MAXENT-NSOM. However, we restricted the calculations to what we have called the second-order approximation in relaxation theory. In SOART the equations of evolution are instantaneous in time (memoryless). Furthermore, we have only introduced the density of carriers and the linear momentum density as basic variables to describe the evolution of the carrier system. As noted elsewhere, ${ }^{18}$ this implies a truncation procedure (higherorder tensorial fluxes are neglected) valid in the longwavelength limit, as is the case in the hydrodynamical analysis we performed. In this way we are able consider the motion of the carrier density. A complete hydrodynamical analysis would also incorporate as basic vari- ables the density of the energy and its flux, which we have associated with the propagation of damped thermal waves. $^{3}$ Both types of motion, material and thermal, are coupled through cross-kinetic terms. Our analysis here neglected such cross-transport effects, concentrating attention only on the movement of mass (the evolution in space and the time of the charge density).

As shown in Sec. II the motion of the carrier density is governed by an equation describing damped waves. It is an equation of the telegraphist type [hyperbolic partial differential equation; cf. Eq. (13)], where $c$ is the velocity of propagation, and $D$ is a diffusion coefficient (setting $c$ going to infinity, but $D$ finite, and neglecting the term with coefficient $A$, we obtain the equivalent of Fick's diffusion equation). $D$ is the ambipolar diffusion coefficient, defined at the microscopic mechanostatistical level by Eq. (15b). In nonstationary conditions the dependence of $D$ on time arises out of the fact that it is, in the MAXENT-NSOM and SOART, dependent on the instantaneous nonequilibrium state of the system. In Ref. 31 ambipolar diffusion effects in photoexcited plasmas in semiconductors are analyzed in detail and a comparison with experiment is presented.

Our analysis of the charge propagation done in Sec. II allowed us - through an analysis of the eigenvalues of the equation of evolution in the steady state [cf. Eq. (6)] - to characterize four types of elementary excitations (the hydrodynamic modes are obtained, we recall, in the limit of long wavelengths, i.e., small $Q$ ). Two are associated with single-particle excitations of electrons and holes; the other two are collective excitations. These collective excitations are plasma waves of two kinds: (i) an optical plasmon corresponding to a collective movement associated with the relative coordinates of the electron-hole pairs and the highest in frequency of all four excitations, and the pair interacting through the bare Coulomb interaction; and (ii) an acoustic plasmon corresponding to a collective movement associated with the centers of mass of the electron-hole pairs, which is low in frequency and superposed with the continuum of quasiparticle excitations and, thus, involving pairs interacting through a screened Coulomb interaction. As noted in Sec. I, these two types of collective excitation were predicted by Pines, and experimentally observed by Pinczuk, Shah, and Wolff in the case of the photoexcited plasma in GaAs.

We have derived expressions for the lifetime of both plasma modes [cf. Eqs. (12)], and all the excitations are brought into evidence in the Raman spectrum, calculated in Sec. III, since each type of excitation contributes to the scattering of photons, producing a characteristic band in the spectrum: the lowest in frequency is the one due to scattering by single-hole excitations, followed, with increasing values in frequency, by those due to scattering by acoustic plasmons, single electrons, and optical plasmons.

\section{ACKNOWLEDGMENTS}

We acknowledge the financial support to our Group provided through the years by the State of São Paulo Research Foundation (FAPESP) and the Brazilian National Research Council (CNPq). 
${ }^{1}$ A. Elci, M. O. Scully, A. L. Smirl, and J. C. Matter, Phys. Rev. B 16, 191 (1977); J. Collet, T. Amand, and M. Pugnet, Phys Lett. 96A, 368 (1983); P. Motisuke, C. A. Arguello, and R. Luzzi, Solid State Commun. 23, 617 (1977).

${ }^{2}$ A. C. Algarte, A. R. Vasconcellos, and R. Luzzi, in Proceedings of the 18th International Conference on the Physics of Semiconductors, edited by O. Engström (World Scientific, Singapore, 1986), Vol. 2, pp. 1295-1298.

${ }^{3}$ A. R. Vasconcellos, R. Luzzi, D. Jou, and J. Casas-Vazquez, following paper, Phys. Rev. B 52, 5030 (1995).

${ }^{4} \mathrm{~S}$. de Groot and P. Mazur, Nonequilibrium Thermodynamics (North-Holland, Amsterdam, 1962).

${ }^{5}$ L. S. Garcia-Colin and F. J. Uribe, J. Non-Equilib. Thermodyn. 16, 89 (1991).

${ }^{6}$ I. Müller and T. Ruggeri, Extended Thermodynamics (Springer, Berlin, 1993).

${ }^{7}$ D. Jou, J. Casas-Vazquez, and G. Lebon, Extended Irreversible Thermodynamics (Springer, Berlin, 1993).

${ }^{8}$ J. C. Maxwell, Philos. Trans. R. Soc. London 157, 49 (1867), reprinted in The Collected Papers of J. C. Maxwell, edited by W. R. Niven (Dover, New York, 1965).

${ }^{9}$ H. Grad, in Thermodynamics of Gases, Handbuch der Physik, edited by S. Flügge (Springer, Berlin, 1958), Vol. 12.

${ }^{10}$ R. M. Velasco and L. S. Garcia-Colin, J. Non-Equilib. Thermodyn. 18, 157 (1993).

${ }^{11} \mathrm{R}$. Zwanzig, in Perspectives in Statistical Physics, edited by $\mathbf{H}$. R. Ravechè (North-Holland, Amsterdam, 1981).

${ }^{12}$ R. Luzzi and A. R Vasconcellos, Fortschr. Phys. 38, 887 (1990).

${ }^{13}$ E. T. Jaynes, in Frontiers of Nonequilibrium Statistical Physics, edited by G. T. Moore and M. O. Scully (Plenum, New York, 1986); Ann. Rev. Phys. Chem. 31, 579 (1980).

${ }^{14}$ E. T. Jaynes, in E. T. Jaynes Papers on Probability, Statistics, and Statistical Physics, edited by D. Rosenkrantz (Reidel, Dordrecht, 1983).

${ }^{15}$ P. W. Anderson, Phys. Today 45 (1), 9 (1992).

${ }^{16}$ E. T. Jaynes, Proc. IEEE 70, 939 (1982); Phys. Rev. 106, 620 (1957); 108, 171 (1957).

${ }^{17}$ W. T. Grandy, Foundations of Statistical Mechanics (Reidel, Dordrecht, 1986), Vols. I and II.
${ }^{18}$ A. R. Vasconcellos, R. Luzzi, and L. S. Garcia-Colin, Phys. Rev. A 43, 6622 (1991); 43, 6633 (1991).

${ }^{19}$ L. S. Garcia-Colin, A. R. Vasconcellos, and R. Luzzi, J. NonEquilib. Thermodyn. 19, 24 (1993): J. Kestin Memorial Issue; R. Luzzi and A. R. Vasconcellos (unpublished).

${ }^{20}$ R. E. Nettleton, J. Phys. A 22, 5281 (1989); 42, 4622 (1990).

${ }^{21}$ R. E. Nettleton, J. Chem. Phys. 93, 8247 (1990); 97, 8815 (1992); S. Afr. J. Phys. 14, 27 (1991).

${ }^{22}$ L. Lauck, A. R. Vasconcellos, and R. Luzzi, Physica A 168, 789 (1990).

${ }^{23}$ A. R. Vasconcellos, R. Luzzi, and L. S. Garcia-Colin (unpublished).

${ }^{24}$ A. C. Algarte and R. Luzzi, Phys. Rev. B 27, 7563 (1983); V. N. Freire, A. R. Vasconcellos, and R. Luzzi, ibid. 39, 13264 (1989); see also Ref. 31.

${ }^{25}$ D. N. Zubarev, Neravnovesnaia Statisticheskaia Thermodinamika (Nauka, Moscow, 1971) [English translation: Nonequilibrium Statistical Thermodynamics (Consultants Bureau, New York, 1974)]; in Reviews of Science and Technology: Modern Problems of Mathematics, edited by R. B. Gamkreludze (Nauka, Moscow, 1980), Vol. 15 [English translation in J. Sov. Math. 16, 1509 (1981)].

${ }^{26}$ D. Pines, Can. J. Phys. 34, 1379 (1956); P. Nozières and D. Pines, Phys. Rev. 109, 741 (1958); 109, 762 (1985); 109, 1062 (1958); D. Pines and J. R. Schrieffer, ibid. 124, 1387 (1961).

${ }^{27}$ A. Pinczuk, J. Shah, and P. A. Wolff, Phys. Rev. Lett. 47, 1487 (1981).

${ }^{28}$ P. M. Platzmann, Phys. Rev. 39, A379 (1965).

${ }^{29}$ G. Vignale and K. S. Singwi, Phys. Rev. B 31, 245 (1985).

${ }^{30}$ A. W. Overhauser and J. Apple, Phys. Rev. B 26, 507 (1982); 29, 1069 (1984).

${ }^{31}$ A. C. Algarte, A. R. Vasconcellos, and R. Luzzi, Phys. Rev. B 48, 10873 (1993).

${ }^{32}$ A. C. Algarte, A. R. Vasconcellos, and R. Luzzi, Phys. Status Solidi B 173, 487 (1992).

${ }^{33}$ T. Tomé, A. R.Vasconcellos, and R. Luzzi, Physica B 144, 376 (1987).

${ }^{34}$ P. A Wolff, in Raman Scattering Spectra in Solids I, edited by G. B. Wright (Springer, New York, 1969), pp. 273-284. 